\title{
Single Strontium Rydberg Ion Confined in a Paul Trap
}

\author{
Gerard Higgins, ${ }^{1,2}$ Weibin Li, ${ }^{3}$ Fabian Pokorny, ${ }^{1}$ Chi Zhang, ${ }^{1}$ Florian Kress, ${ }^{2}$ Christine Maier, ${ }^{2}$ \\ Johannes Haag, ${ }^{2}$ Quentin Bodart, ${ }^{1}$ Igor Lesanovsky, ${ }^{3}$ and Markus Hennrich ${ }^{1, *}$ \\ ${ }^{1}$ Department of Physics, Stockholm University, 10691 Stockholm, Sweden \\ ${ }^{2}$ Institut für Experimentalphysik, Universität Innsbruck, 6020 Innsbruck, Austria \\ ${ }^{3}$ School of Physics and Astronomy, University of Nottingham, Nottingham NG7 2RD, United Kingdom \\ and Centre for the Mathematics and Theoretical Physics of Quantum Non-equilibrium Systems, \\ University of Nottingham, Nottingham NG7 2RD, United Kingdom \\ (Received 8 December 2016; revised manuscript received 1 April 2017; published 7 June 2017)
}

\begin{abstract}
Trapped Rydberg ions are a promising new system for quantum information processing. They have the potential to join the precise quantum operations of trapped ions and the strong, long-range interactions between Rydberg atoms. Combining the two systems is not at all straightforward. Rydberg atoms are severely affected by electric fields which may cause Stark shifts and field ionization, while electric fields are used to trap ions. Thus, a thorough understanding of the physical properties of Rydberg ions due to the trapping electric fields is essential for future applications. Here, we report the observation of two fundamental trap effects. First, we investigate the interaction of the Rydberg electron with the trapping electric quadrupole fields which leads to Floquet sidebands in the excitation spectra. Second, we report on the modified trapping potential in the Rydberg state compared to the ground state that results from the strong polarizability of the Rydberg ion. By controlling both effects we observe resonance lines close to their natural linewidth demonstrating an unprecedented level of control of this novel quantum platform.
\end{abstract}

DOI: 10.1103/PhysRevX.7.021038

\section{INTRODUCTION}

Trapped ions are one of the most mature implementations of a quantum computer. The trapped ion approach has set several benchmarks with qubit lifetimes up to minutes [1], entanglement operations with error probabilities smaller than $10^{-3}[2,3]$, and with up to 14 entangled qubits [4]. Trapped ions also assume a leading role in the implementation of quantum algorithms [5-8], quantum error correction [9-11], and quantum simulations [12-15].

The standard method to realize quantum information processing with trapped ions uses the common motion for entanglement operations between the ion qubits [16]. A current limitation of trapped ion quantum computation is the limited storage capacity, as it becomes more difficult to perform entanglement operations in large ion crystals due to the increasingly complex motional mode structure. Possible schemes to reach larger quantum systems include segmented ion traps [17], ion-photon networks [18], and trapped Rydberg ions $[19,20]$.

\footnotetext{
*markus.hennrich@fysik.su.se
}

Published by the American Physical Society under the terms of the Creative Commons Attribution 4.0 International license. Further distribution of this work must maintain attribution to the author(s) and the published article's title, journal citation, and DOI.
Subject Areas: Atomic and Molecular Physics,

Quantum Information
Trapped Rydberg ions are a novel quantum system. Here, the outermost electron of an ion is excited into Rydberg states far away from the atomic core. Pairs of Rydberg ions are envisaged to couple strongly via electric dipole-dipole interaction. Quantum gates using Rydberg interactions may be realized independently of the ionic vibrational modes [21]; thus, Rydberg ions may be used in larger ion crystals for entanglement operations [19,22]. A similar entanglement method has been demonstrated with neutral atoms [23-25]. In this sense trapped Rydberg ions promise to join the advantages of both technologies: they combine the strong dipolar interaction between Rydberg atoms with the precise quantum control and long storage times of trapped ions.

Combining these systems is by no means trivial. The trapping electric fields will need to stay active while exciting ions to Rydberg states, otherwise the strong Coulomb repulsion will quickly push the ions apart. Rydberg atoms, however, are extremely sensitive to electric fields, which cause Stark shifts and may even ionize the Rydberg electron. In neutral Rydberg atom experiments any electric fields are carefully compensated and so it seems at best far-fetched to excite an ion trapped in strong electric fields to a Rydberg state. Remarkably, trapped Rydberg ions have been realized [26] using single-photon excitation of ${ }^{40} \mathrm{Ca}^{+}$ions with vacuum ultraviolet laser light at $122 \mathrm{~nm}$. Also, selective manipulation of the ground state was combined with optical pumping via the Rydberg state [27]. 
Here, we report on a two-photon Rydberg excitation of ${ }^{88} \mathrm{Sr}^{+}$ions. The wavelengths we use for Rydberg excitation (243 and $309 \mathrm{~nm}$ ) are significantly easier to handle than the vacuum ultraviolet light used before [26]. In particular, the laser photons contain significantly lower energy; thus, no surface charging of the trap electrodes is observed. Also, in a two-photon excitation the lasers can be sent from opposite sides, which puts the ions in an effective Lamb-Dicke regime and thus avoids Doppler broadening of the resonances. These advantages allow us to achieve an unparalleled level of control and to investigate the fundamental physical effects of the trap on Rydberg ions.

Modifications of the Rydberg properties due to the strong electric fields of the Paul trap have been predicted $[19,20]$. One of the expected effects is that the Rydberg electron will interact with the trapping electric quadrupole fields. While Rydberg $S_{1 / 2}$ and $P_{1 / 2}$ states do not possess quadrupole moments due to their symmetry, and thus do not interact with the quadrupole fields, higher angular momentum states will be affected. We experimentally investigate this fundamental effect, explore the different behavior of Rydberg $S_{1 / 2}$ and $D_{3 / 2}$ states, and compare the experimental results to theoretical simulations.

A further novel property of Rydberg-excited ions is that their trapping potential is modified compared to their ground state, which can, e.g., induce structural phase transitions in an ion crystal [28]. This effect is caused by the strong polarizability of the Rydberg state, which becomes polarized in the trapping electric fields. Depending on the sign of the polarizability, the induced dipole either weakens or increases the trapping electric field experienced by the ion and thus modifies the confining potential. We observe a first signature of this effect investigating a single Rydberg resonance. In particular, we see the modified trapping potential as a line broadening for a Doppler-cooled ion with a thermal population distribution, as compared to a sideband-cooled ion where most of the population resides in the motional ground state.

\section{EXPERIMENTAL SYSTEM}

A single ${ }^{88} \mathrm{Sr}^{+}$ion is confined in a linear Paul trap and excited to Rydberg $S_{1 / 2}$ and $D_{3 / 2}$ states by a two-photon process, as shown in Fig. 1. 243-nm laser light drives the first step from the metastable $4 D_{3 / 2}$ state to $6 P_{1 / 2}$, while tunable laser light at 304-309 nm excites the second step to reach Rydberg $S_{1 / 2}$ and $D_{3 / 2}$ states.

During Rydberg excitation the ion is confined in the electric fields of a linear Paul trap. Note that the Rydberg ions do not get doubly ionized by the trapping electric fields, as ions are generally held at the null of the electric quadrupole fields, at least given that stray electric fields leading to micromotion of the ion are properly compensated. For further details on the experimental setup and the micromotion compensation, see Appendixes A and B. (a)

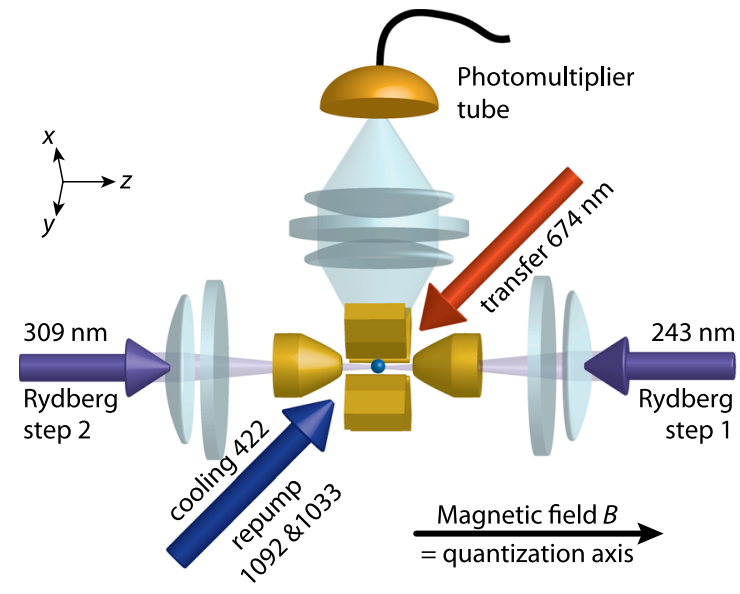

(b)

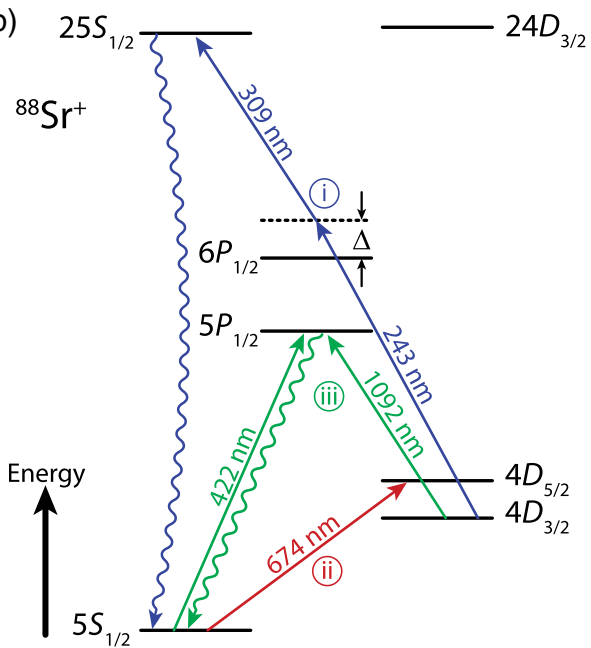

FIG. 1. Experimental setup. (a) An ion is trapped in a linear Paul trap and manipulated by laser beams for Doppler cooling and fluorescence detection (422 nm), repumping (1092 and $1033 \mathrm{~nm})$, electron shelving (674 nm), and Rydberg excitation (243 and $309 \mathrm{~nm})$. A magnetic field with $B=(0.3564 \pm 0.0008) \mathrm{mT}$ defines the quantization axis and is oriented parallel to the trap axis and the Rydberg-excitation lasers. (b) Energy level scheme of ${ }^{8} \mathrm{Sr}^{+}$and detection sequence of successful Rydberg excitation. Before Rydberg excitation the Doppler-cooled ion is initialized in the metastable $4 D_{3 / 2}$ state via optical pumping. (i) The Rydberg-excitation lasers couple the initial $4 D_{3 / 2}$ state to a Rydberg $S_{1 / 2}$ or $D_{3 / 2}$ state, which then quickly decays in multiple steps to the $5 S_{1 / 2}$ ground state with $95 \%$ probability in $<\sim 20 \mu \mathrm{s}$. (ii) Rydberg-excited population which subsequently decayed to the ground state is transferred to the metastable $4 D_{5 / 2}$ state, allowing fluorescence detection to distinguish between successful Rydberg excitations (population in $4 D_{5 / 2} \rightarrow$ no fluorescence) and cases with no Rydberg excitations (population in $4 D_{3 / 2} \rightarrow$ fluorescence) in the final step (iii). This sequence is typically repeated 100 times for each data point. 


\section{IONIC RYDBERG STATES IN A LINEAR PAUL TRAP}

In the following, we provide the theoretical background concerning the Rydberg excitation of a strontium ion held in a linear Paul trap. This allows us to undertake a comparison between experimentally obtained and theoretically calculated excitation spectra. Specifically, it enables us to identify and quantify the influence of the trapping fields on the Rydberg-ion level structure.

The electric potential of the Paul trap reads

$$
\Phi(\mathbf{r}, t)=\alpha \cos \Omega t\left(x^{2}-y^{2}\right)-\beta\left(x^{2}+y^{2}-2 z^{2}\right),
$$

where $\alpha$ and $\beta$ are electric field gradients and $\Omega$ is the frequency of the radio frequency electric field. In contrast to low-lying states, the weakly bound Rydberg electron can exhibit a sizable coupling to the trap electric fields. To illustrate this, we write the coupling Hamiltonian $H_{e Q}(\mathbf{r}, t)$ as [20]

$$
\begin{aligned}
H_{e Q}(\mathbf{r}, t)= & e r^{2}\left[-2 \sqrt{\frac{\pi}{5}} \beta Y_{2}^{0}(\theta, \phi)\right. \\
& \left.-\sqrt{\frac{8 \pi}{15}} \alpha \cos \Omega t Y_{2}^{2}(\theta, \phi)+\text { H.c. }\right],
\end{aligned}
$$

where $\theta$ and $\phi$ are polar and azimuthal angles with respect to the trap axis $z$ and $Y_{l}^{m}(\theta, \phi)$ are spherical harmonics.

For a Rydberg state $\left|n L J m_{J}\right\rangle$, with $n, L, J$ the principal, angular, and total angular quantum numbers, and $m_{J}$ the projection of $J$ on the quantization axis (along the trap $z$ axis), the quadrupole coupling is nonzero when $J>1 / 2$, while it vanishes when $J=1 / 2$ due to selection rules. This shows that there is no first-order effect for Rydberg $S_{1 / 2}$ states ( $L=0$ and $J=1 / 2$ ). However, the coupling becomes significant in Rydberg $D_{J}$ states $(J=3 / 2$ or $J=5 / 2$ ), as illustrated in Fig. 2. Specifically, for $J=3 / 2$ and magnetic field $B \| z$, the static part of the trap potential causes an energy shift to state $\left|n D \frac{3}{2} m_{J}\right\rangle$,

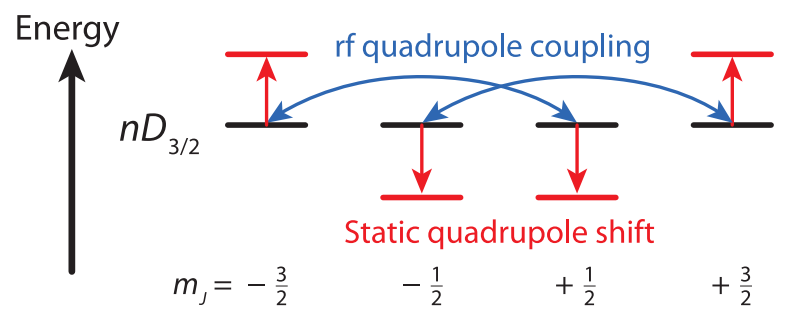

FIG. 2. Rydberg electron-quadrupole field interaction. Quadrupolar energy shifts and rf coupling due to the Rydberg electronquadrupole field interaction $H_{e Q}$ for a Rydberg $D_{3 / 2}$ state.

$$
E_{s}=\frac{2}{5}(-1)^{\left|m_{J}\right|-1 / 2} \beta Q_{3 / 2},
$$

where $Q_{J}=-e\left\langle n D J\left|r^{2}\right| n D J\right\rangle$ denotes the corresponding quadrupole transition moment. For $n \gg 1$, its approximate value is $Q_{J} \approx\left[e a_{0}^{2} n^{2}\right] /\left[2\left(2 Z_{e}+1\right)\right]\left[5 n^{2}+1-3 L(L+1)\right]$, with core charge $Z_{e}=2$ and Bohr radius $a_{0}$. From Eq. (3) it follows that the energy shifts for $\left|m_{J}\right|=1 / 2$ and $\left|m_{J}\right|=$ $3 / 2$ have the same strength but opposite signs, as depicted in Fig. 2.

The rf-dependent part of Hamiltonian Eq. (2), on the other hand, couples different Zeeman states with $\Delta m_{J}=$ \pm 2 ; see Fig. 2. As the rf drive is much smaller than the finestructure splitting $(\Omega / 2 \pi \sim \mathrm{MHz})$, we restrict the coupling to states within the same Zeeman manifold (for states with identical quantum numbers $n, L$, and $J$ ). The rf-dependent part of Eq. (2) then assumes the form

$$
H_{\mathrm{rf}}=\hbar C \cos \Omega t \sum_{m_{J}=1 / 2}^{3 / 2}\left[\left|n L J\left(m_{J}-2\right)\right\rangle\left\langle n L J m_{J}\right|+\text { H.c. }\right] \text {, }
$$

where the constant $C=-2 Q_{J} \alpha / 5 \sqrt{3} \hbar$ is the effective Rabi frequency of the rf field.

In the current experiment, typical trap parameters are $\alpha \approx 3 \times 10^{8} \mathrm{Vm}^{-2}, \beta \approx 6 \times 10^{5} \mathrm{Vm}^{-2}$, and $\Omega \approx 2 \pi \times 18 \mathrm{MHz}$. This yields a static frequency shift $\left|E_{s}\right| / \hbar \approx 2 \pi \times 43 \mathrm{kHz}$ and effective Rabi frequency $C \approx 2 \pi \times 12 \mathrm{MHz}$ in the Rydberg state $\left|24 D \frac{3}{2} m_{J}\right\rangle$. Note that the latter is comparable with the $\mathrm{rf}$ drive, $C \sim \Omega$. An emerging feature is that Floquet sidebands will be populated [29], as the rotatingwave approximation is not applicable in the quadrupole coupling Hamiltonian $H_{\mathrm{rf}}$.

All subsequent calculations of the Rydberg spectra (shown in Figs. 3 and 4) are performed with coupling Hamiltonian of the form Eq. (2). We take into account the experimental trap geometry and laser parameters (see Appendix D for the laser-ion interaction). The laser excitation dynamics is described through a quantum master equation.

\section{RYDBERG ELECTRON-QUADRUPOLE FIELD INTERACTION}

According to the previous considerations, Rydberg $S_{1 / 2}$ states are expected to not interact with the trapping electric quadrupole fields; thus, we expect an excitation spectrum with a simple structure. The experimental results for $25 S_{1 / 2}$ are shown in Fig. 3. Peaks in the $4 D_{5 / 2}$ population result from the excitation of the ion to $25 S_{1 / 2}$ Rydberg state at excitation energy $(88081.84 \pm 0.04) \mathrm{cm}^{-1}$ with respect to the ground state of $\mathrm{Sr}^{+}$consistent with values reported in Ref. [30]. Depending on the laser polarizations used, one, two, or four peaks are observed. The observed resonance lines can be easily explained by the Zeeman splitting in the 

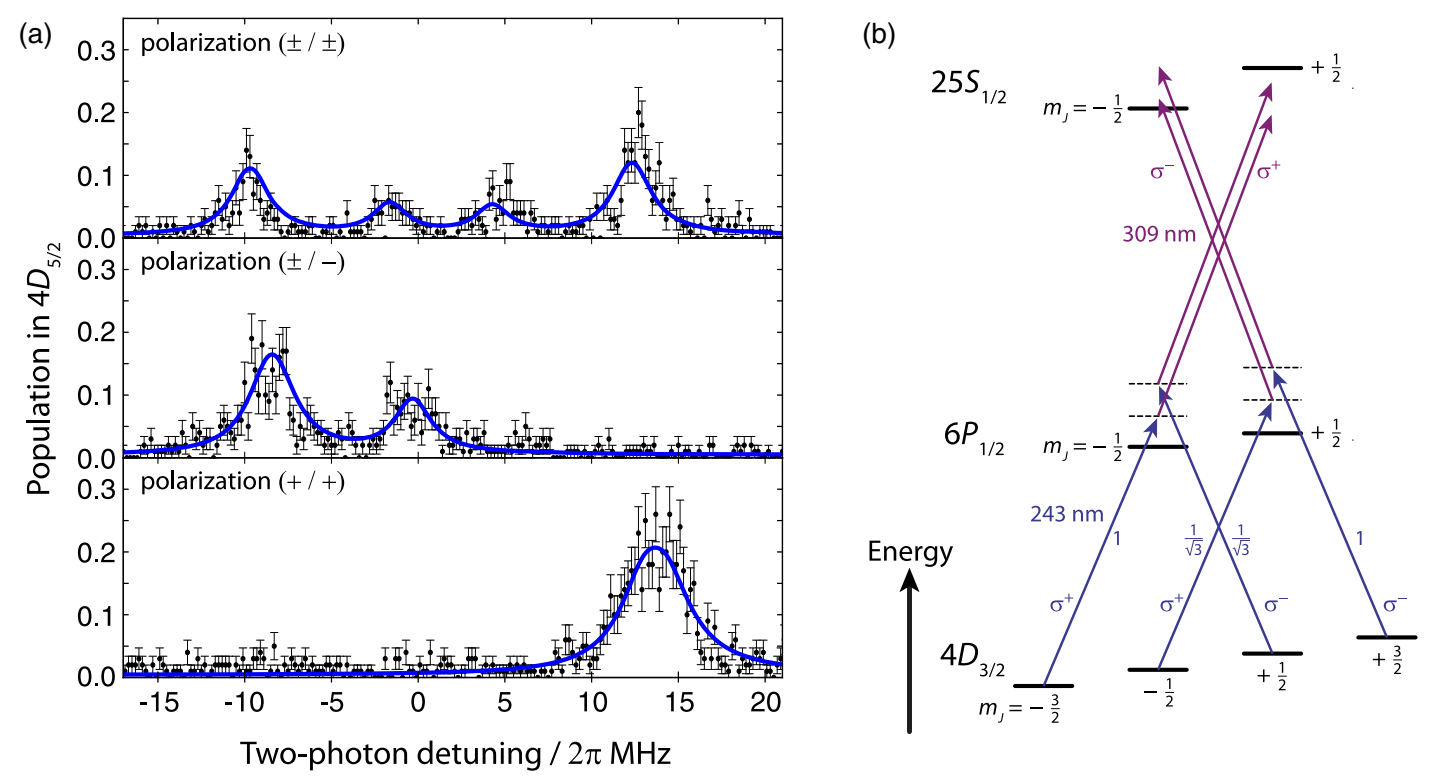

FIG. 3. Zeeman splitting in Rydberg $S_{1 / 2}$ states. (a) Two-photon excitation spectra to state $25 S_{1 / 2}$. The frequency of the second Rydberg excitation laser at $309 \mathrm{~nm}$ is scanned while the frequency of the first Rydberg excitation laser at $243 \mathrm{~nm}$ is kept $2 \pi \times 160 \mathrm{MHz}$ blue detuned from the intermediate state resonance. The two-photon detuning is defined as the sum of the two UV-photon frequencies minus the frequency difference of the $25 S_{1 / 2}$ and $4 D_{3 / 2}$ states in the absence of a magnetic field $\omega_{243}+\omega_{309}-(1 / \hbar)\left(E_{25 S_{1 / 2}}-E_{4 D_{3 / 2}}\right)$. Successful Rydberg excitation is signaled by a high probability for shelving the ion to state $4 D_{5 / 2}$. The excitation spectrum shows four resonance peaks for both lasers in an equal superposition of $\sigma^{+}$and $\sigma^{-}$polarization [denoted as $( \pm / \pm)$], two peaks when the second step 309 -nm laser is changed to $\sigma^{-}( \pm /-)$, and a single peak when both lasers are $\sigma^{+}(+/+)$. Black dots are the measured data points with error bars due to quantum projection noise, blue lines are the simulated Rydberg-excitation spectra (Rabi frequencies $\Omega_{243}=2 \pi \times 0.47 \mathrm{MHz}, \Omega_{309}=2 \pi \times 49 \mathrm{MHz}$, dephasing of the Rydberg state $\delta \omega_{25 S}=2 \pi \times 2.2 \mathrm{MHz}$ ). Off-resonant scattering from the intermediate state and spontaneous decay from the initial $4 D_{3 / 2}$ state each contribute to the background signal. (b) Allowed transitions for Rydberg excitation to $25 S_{1 / 2}$. The Rydberg-excitation beams are aligned with the direction of the applied magnetic field at the position of the ion; thus, electric dipole transitions which preserve the magnetic quantum number ( $\pi$ transitions) are not excited. With this constraint, only four nondegenerate transitions between the $4 D_{3 / 2}$ and $25 S_{1 / 2}$ Zeeman sublevels remain. As the frequency of the 309-nm laser is scanned, each of the four transitions comes into resonance at a different frequency.

applied magnetic field of $B=(0.3564 \pm 0.0008) \mathrm{mT}$, where each of the four equally populated initial Zeeman levels couples to exactly one Rydberg level; see Fig. 3(b). The simulation results corroborate this explanation. Relative amplitudes agree with the difference in Rabi frequencies due to the respective Clebsch-Gordan coefficients. Thus, the spectroscopy of the Rydberg $S$ state can be fully explained by the Zeeman effect in the applied magnetic field. No effect of the trapping electric quadrupole fields on the $S$-state Rydberg electron is observed.

The effect of the Rydberg electron-quadrupole field interaction becomes evident in the excitation spectra of the $24 D_{3 / 2}$ Rydberg state [experimental excitation energy (88 101.36 \pm 0.04$) \mathrm{cm}^{-1}$ ]; see Fig. 4. We observe a multitude of resonances with Floquet sidebands caused by the rf coupling between Zeeman sublevels within the $24 D_{3 / 2}$ manifold. The simulation matches the experimental data when the Rydberg electron-quadrupole field interaction [Eq. (2)] is included. Also, matching the positions of the ac-Stark shifted resonances in the simulation and the experimental data allows the Rabi frequency of the $309-\mathrm{nm}$ laser to be determined to within $10 \%$. Smaller deviations between the experimental data and the theoretical model may be explained by experimental imperfections and ion motion, such as imperfect polarization, motional sideband transitions, thermal broadening, and micromotion effects, which are not included in the simulation.

The observed resonance lines can be explained in simple terms as depicted in Fig. 4(b). Because of the rf coupling of the Rydberg electron-quadrupole field interaction, sidebands at $\pm \Omega$ relative to neighboring Zeeman levels $\left(\Delta m_{J}= \pm 2\right)$ become visible. For high Rabi frequencies the lines are offset by ac-Stark shifts. The resonance frequencies can be identified by a diagonalization of the coupling Hamiltonian within the $24 D_{3 / 2}$ manifold; see Appendix E. For instance, for $\sigma^{-} / \sigma^{+}$polarizations for the first or second Rydberg-excitation steps and the Rabi frequencies, as in the matching simulation, we obtain four eigenfrequencies at $2 \pi\{5.8,9.2,14.1,29.0\} \mathrm{MHz}$ detuning with corresponding eigenstates 

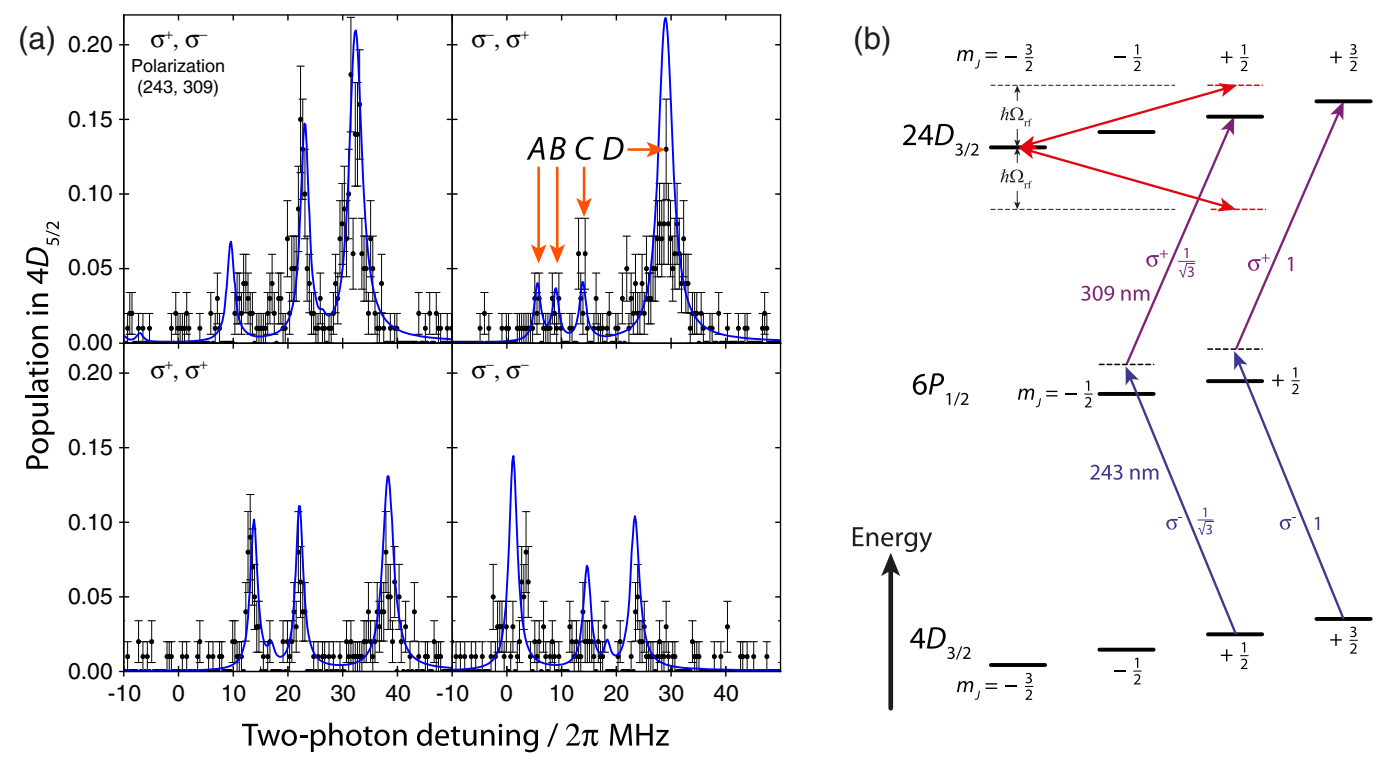

FIG. 4. Rydberg electron-quadrupole field interaction for Rydberg $D_{3 / 2}$ states. (a) Excitation spectra and simulation results for $24 D_{3 / 2}$. The frequency of the second Rydberg excitation laser at $309 \mathrm{~nm}$ is scanned while the frequency of the first Rydberg excitation laser at $243 \mathrm{~nm}$ is kept $2 \pi \times 160 \mathrm{MHz}$ blue detuned from the intermediate state resonance. The two-photon detuning is defined as the sum of the two UV-photon frequencies minus the frequency difference of the $24 D_{3 / 2}$ and $4 D_{3 / 2}$ states in the absence of a magnetic field, trap effects, and ac-Stark effects $\omega_{243}+\omega_{309}-(1 / \hbar)\left(E_{25 S_{1 / 2}}-E_{4 D_{3 / 2}}\right)$. The trapping parameters are $\left\{\omega_{\text {axial }}, \omega_{\text {radial } 1}, \omega_{\text {radial } 2}\right\}=$ $2 \pi\{(254 \pm 3),(600 \pm 10),(760 \pm 10)\} \mathrm{kHz}$, radio frequency drive $\Omega=2 \pi \times 18.153 \mathrm{MHz}$. The experimental data (error bars due to quantum projection noise) largely match the simulation results (dark blue line) when the Rydberg electron-quadrupole field interaction is taken into account (simulation parameters: Rabi frequencies $\Omega_{243}=2 \pi \times 0.09 \mathrm{MHz}, \Omega_{309}=2 \pi \times 135 \mathrm{MHz}$, dephasing of the Rydberg state $\delta \omega_{24 D}=2 \pi \times 4.1 \mathrm{MHz}$ ). (b) Energy level scheme explaining the rf sidebands in the excitation spectra. Sidebands appear at \pm the rf trapping frequency $\Omega$ with respect to a neighboring Zeeman state $\Delta m_{J}= \pm 2$. The resonances are additionally shifted due to the ac-Stark effect by the Rydberg excitation laser at $309 \mathrm{~nm}$.

$$
\begin{aligned}
& |A\rangle=+0.72\left|\frac{1}{2}, 0\right\rangle-0.69\left|\frac{3}{2},+1\right\rangle, \\
& |B\rangle=-0.21\left|\frac{3}{2}, 0\right\rangle+0.98\left|\frac{1}{2},+1\right\rangle, \\
& |C\rangle=+0.69\left|\frac{1}{2}, 0\right\rangle+0.72\left|\frac{3}{2},+1\right\rangle, \\
& |D\rangle=+0.97\left|\frac{3}{2}, 0\right\rangle+0.22\left|\frac{1}{2},+1\right\rangle,
\end{aligned}
$$

where we use $\left|m_{J}, n\right\rangle=\left|24 D \frac{3}{2} m_{J}, n\right\rangle$, with $n$ being the number of quadrupole excitations of the Floquet sidebands. The corresponding states $|A\rangle-|D\rangle$ are marked in the top right-hand panel of Fig. 4. In these states, the "absorbing" states $\left|m_{J},-1\right\rangle$ have negligible contributions, since the Rydberg electron-quadrupole field interaction is further detuned; see Fig. 4(b).

\section{MODIFIED TRAPPING POTENTIAL IN THE RYDBERG STATE}

The strong polarizability of the Rydberg state is expected to modify the effective trapping potential of the ion. The change in the radial trapping potential of the Rydberg state compared to lower-lying states is [28]

$$
V_{\text {add }}=-\left(\alpha^{2}+2 \beta^{2}\right) \mathcal{P}_{n, L} \rho^{2} \approx-\alpha^{2} \mathcal{P}_{n, L} \rho^{2},
$$

where $\rho$ is the radial center-of-mass coordinate of the ion and $\mathcal{P}_{n, L}$ is the polarizability of the Rydberg state with quantum numbers $n$ and $L$. The polarizability scales as $\sim n^{7}$; thus, the influence of $V_{\text {add }}$ should increase for higher Rydberg states.

We now investigate the effect of the modified trapping potential by analyzing the Rydberg-excitation spectrum of state $42 S_{1 / 2}$ [experimental excitation energy (88680.74士 $\left.0.04) \mathrm{cm}^{-1}\right]$. The controlled preparation of the ion in a single Zeeman sublevel combined with driving only a single transition in the Rydberg excitation leads to the observation of a single Rydberg resonance, as depicted in Fig. 5(a). The details on state preparation and detection of Rydberg excitation in this context are given in Appendix C. After Doppler cooling we observe a linewidth of $2 \pi(1.4 \pm 0.1) \mathrm{MHz}$, which is significantly broader than the expected natural linewidth calculated from quantum defect theory of $2 \pi \times 39 \mathrm{kHz}$. However, with radial sideband cooling the observed linewidth is reduced to $2 \pi \times 300 \mathrm{kHz}$. This sideband-cooled linewidth can be fully explained by natural and laser linewidths $(\approx 2 \pi \times 200 \mathrm{kHz})$.

Commonly, one would suspect the broadening to be caused by the Doppler effect; however, we do not observe a 

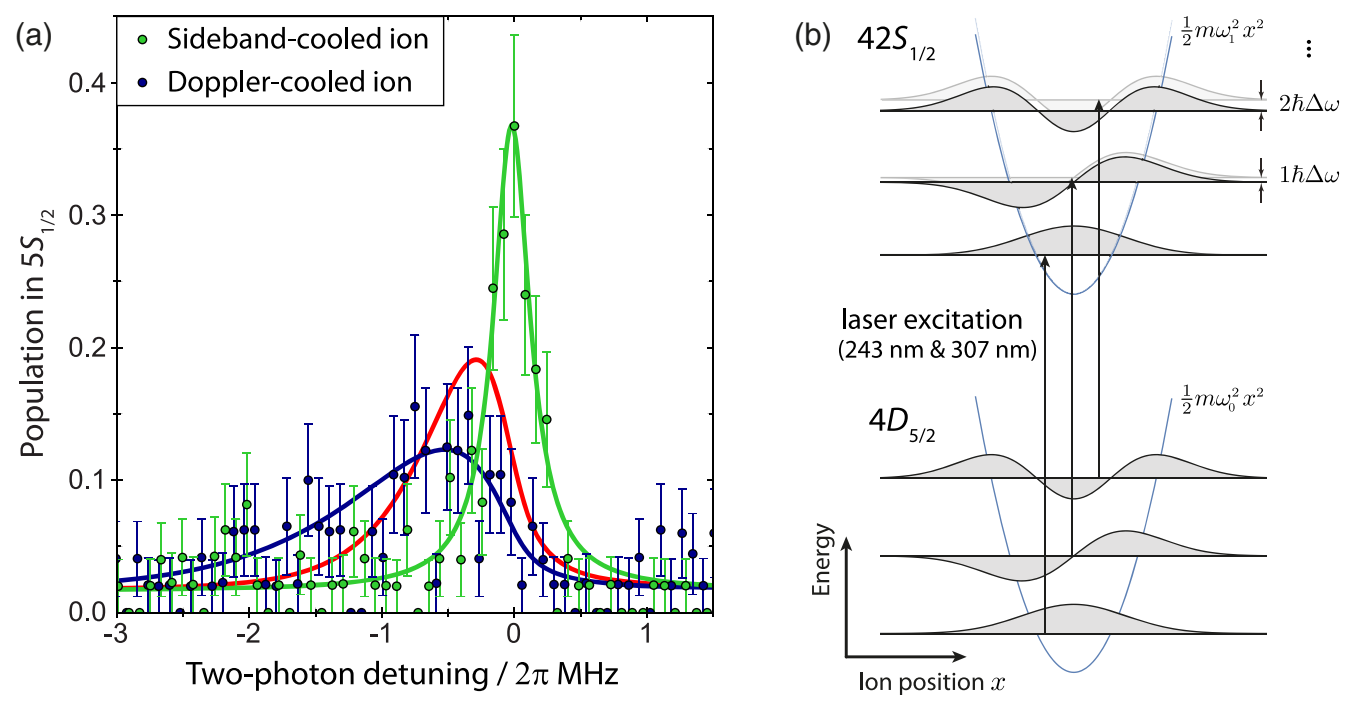

FIG. 5. Modified Rydberg trapping potential. (a) Observation of a single Rydberg resonance starting from the state $4 D_{5 / 2} m_{J}=-\frac{5}{2}$ to the Rydberg state $42 S_{1 / 2} m_{J}=-\frac{1}{2}$. The frequency of the second Rydberg excitation laser at $307 \mathrm{~nm}$ is scanned while the frequency of the first Rydberg excitation laser at $243 \mathrm{~nm}$ is kept $2 \pi \times 20 \mathrm{MHz}$ red detuned from the intermediate state resonance. The two-photon detuning is defined as the sum of the two UV-photon frequencies minus the frequency difference of the $42 S_{1 / 2} m_{J}=-\frac{1}{2}$ and $4 D_{5 / 2} m_{J}=$ $-\frac{5}{2}$ states, $\omega_{243}+\omega_{307}-\frac{1}{\hbar}\left[E\left(42 S_{1 / 2} m_{J}=-\frac{1}{2}\right)-E\left(4 D_{5 / 2} m_{J}=-\frac{5}{2}\right)\right]$. With sideband cooling, we observe a single resonance with linewidth $2 \pi(300 \pm 50) \mathrm{kHz}$, mainly limited by the laser linewidths. With only Doppler cooling, the resonance is shifted lower in energy and has smaller amplitude and asymmetric shape with $\approx 2 \pi(1.4 \pm 0.1) \mathrm{MHz}$ linewidth. The model curves represent a single resonance with $2 \pi(300 \pm 50) \mathrm{kHz}$ linewidth for the sideband-cooled case, and a thermally broadened line with $\Delta \omega=-2 \pi \times 20 \mathrm{kHz}$ (red) $[\Delta \omega=-2 \pi \times 42 \mathrm{kHz}$ (blue)] for the Doppler-cooled case. No fit parameters are used in the model curve of the Doppler-cooled ion resonance. The trapping parameters are $\left\{\omega_{\text {axial }}, \omega_{\text {radial } 1}, \omega_{\text {radial } 2}\right\}=2 \pi\{(872 \pm 5),(1660 \pm 30),(1720 \pm 30)\} \mathrm{kHz}$, radio frequency drive $\Omega=2 \pi \times 18.153 \mathrm{MHz}$. (b) Energy scheme of the motional state during Rydberg excitation. Because of the different trapping potential in the Rydberg state compared to the ground state, the laser excitation is shifted out of resonance for higher motional quantum numbers.

reduced linewidth after axial sideband cooling (along the direction the excitation lasers propagate). Instead, we observe a reduced linewidth after radial sideband cooling (perpendicular to the laser propagation direction). This effect can be explained by the modified trapping potential in the radial directions. The polarizability of $42 S_{1 / 2}$ was calculated to be $\mathcal{P}_{42 S}=2.9 \times 10^{-31} \mathrm{C}^{2} \mathrm{~m}^{2} \mathrm{~J}^{-1}$. This was achieved by diagonalizing the electronic model potential [31], which yields radial wave functions and eigenenergies consistent with the Rydberg series previously measured for $\mathrm{Sr}^{+}$in free space [30]. As a result, the radial trapping frequencies in the Rydberg state $\omega_{1}$ should be smaller than the trapping frequencies in a low-lying state $\omega_{0}$, by $\Delta \omega=$ $\omega_{1}-\omega_{0} \approx-2 \pi \times 20 \mathrm{kHz}$ when the electric field gradient $\alpha=8.3 \times 10^{8} \mathrm{Vm}$. Consequently, the motional energy levels are closer in the Rydberg state than in low-lying states, and the vibrational wave functions are modified; see Fig. 5(b). When the Rydberg-excitation lasers drive transitions between the low-lying state and the Rydberg state, the transition frequency is shifted depending on the initial motional state. In particular, the resonance frequency for phonon number conserving transitions is shifted by $n \Delta \omega$, when the ion has $n$ radial phonons; see Fig. 5(b). Since the wave function in Rydberg and low-lying state are not perfectly orthogonal, the phonon number is not necessarily conserved during Rydberg excitation. Nevertheless, as the trapping potentials in our case are still very similar, so are the vibrational components of the wave functions. Thus, the Franck-Condon factors for the Rydberg excitation are approximately given by Kronecker-delta functions $\delta_{n, m}$ and the population should be mainly transferred to quantum states with the same motional quantum number. In principle, with sufficiently high resolution one should be able to identify individual lines separated by $\Delta \omega$ with relative amplitudes due to the thermal population. In our experiment we cannot resolve the splitting for $42 S_{1 / 2}$ since our lasers are broader than the shifts. Nevertheless, we observe a redshifted, asymmetrically broadened line due to thermal population of the two radial modes of motion; see Fig. 5(a).

We model the asymmetric line shape in Fig. 5(a) taking into account the measured thermal population of the two radial modes after Doppler cooling of $\left\langle n_{x}\right\rangle=14.7 \pm 0.9$ and $\left\langle n_{y}\right\rangle=9.5 \pm 0.8$. The measured data in Fig. 5(a) seem to deviate from this model for the theoretical value of $\Delta \omega=-2 \pi \times 20 \mathrm{kHz}$. If we use $\Delta \omega$ as a free fitting parameter, we reach good agreement for $\Delta \omega=-2 \pi \times 42 \mathrm{kHz}$.

The theory neglects coupling between the ionic motion in the three orthogonal directions. This may account for the 
difference between the theoretical prediction and the experimental results.

\section{CONCLUSION}

We investigate several elementary trap effects of Rydberg ions, which will be essential for future applications in quantum technologies. In particular, we observe the effect of the Rydberg electron-quadrupole field interaction on Rydberg states with different symmetries. While Rydberg $S$ states are unaffected by the Rydberg electronquadrupole field interaction, Rydberg $D$ states interact with the trapping quadrupole fields. In the future, the quadrupole moment of Rydberg $D$ states might lead to new types of interactions like quadrupole-charge, quadrupole-dipole, or quadrupole-quadrupole interactions that could be used for directional coupling in quantum information tasks. Nevertheless, Rydberg $S$ states remain the less intricate system and might thus be easier to control.

Moreover, we are able to deterministically control the initial state for Rydberg excitation, which allows us to investigate a single Rydberg resonance. The modified trapping potential in the Rydberg state has become visible as an increase in the Rydberg resonance linewidth of a Doppler-cooled ion compared to a sideband-cooled ion. As predicted, the large polarizability of the Rydberg states in the electric fields of the Paul trap leads to different trapping potentials in low-lying and Rydberg-excited states, and to broadening of the resonances for a Doppler-cooled ion. The resultant weaker or stronger localization of a Rydberg ion might be used in the future in quantum information processing to cut longer ion chains into smaller subunits for localized quantum operations [32] or for implementing exotic interactions through vibrational mode shaping [33]. A sideband-cooled ion instead is largely unaffected by the modified trapping potential due to its precise localization. Thus, sideband cooling might be required for coherent Rydberg excitation and to avoid unwanted entanglement between the electronic state and the ion motion during Rydberg excitation. Alternatively, microwave-dressed Rydberg states with zero polarizability could be used for realizing Rydberg quantum gates independently of the motional mode structure [21]. Such states would experience the same trapping potential as low-lying states; thus, sideband cooling would not be required. Such Rydberg gates would make trapped Rydberg ions a novel system for quantum information processing.

\section{ACKNOWLEDGMENTS}

We thank Michael Niedermayer for electroplating the trap electrodes, Hsiang-Yu Lo and Jonathan Home for advice on the second harmonic generation of 618-nm light, Yves Colombe for guidance with the fabrication of photonic crystal fibers for UV light, and Ana Predojević for feedback on the manuscript. The experiment started at the University of Innsbruck and moved to Stockholm University. Some of the experimental results were first observed in Innsbruck and have been repeated and confirmed at Stockholm University. The research leading to these results has received funding from the European Research Council under the European Union's Seventh Framework Programme/ERC Grant Agreement No. 279508 (QuaSIRIO). I. L. acknowledges funding from the European Research Council under the European Union's Seventh Framework Programme (FP7/20072013)/ERC Grant Agreement No. 335266 (ESCQUMA) and the H2020-FETPROACT-2014 Grant No. 640378 (RYSQ). W. L. acknowledges access to the University of Nottingham HPC Facility.

\section{APPENDIX A: RYDBERG-EXCITATION LASERS}

The 243-nm laser light for the first Rydberg-excitation step is produced in a commercial system, in which 970-nm infrared laser light from a diode-laser pumped taperedamplifier system is frequency quadrupled to $243 \mathrm{~nm}$. Similar systems are used for the two-photon excitation of hydrogen [34]. Tunable 304-309-nm laser light for the second Rydberg-excitation step is produced in two stages. In the first stage, two infrared photons, from a 1551-nm diode-laser fiber-amplifier system and a tunable 998-1030$\mathrm{nm}$ diode-laser pumped tapered-amplifier system, are combined by sum-frequency generation in a periodically poled lithium niobate crystal $[35,36]$. The resultant $608-$ 618-nm laser light is frequency doubled to produce tunable 304-309-nm laser light, which covers the wavelength range for excitation of Rydberg states from principal quantum number $n=24$ up to the second ionization threshold. The first Rydberg-excitation laser is frequency stabilized to $\approx 2 \pi \times 100-\mathrm{kHz}$ linewidth (in-loop estimate) by locking the 970-nm fundamental to a reference cavity. The second Rydberg-excitation laser is frequency stabilized also to $\approx 2 \pi \times 100-\mathrm{kHz}$ linewidth (in-loop estimate) by referencing 608-618-nm laser light to a cavity and applying feedback to the 998-1030-nm fundamental laser.

The lasers are sent from opposite sides along the trap axis. The counterpropagating beams significantly reduce thermal effects in the two-photon Rydberg excitation. The effective Lamb-Dicke parameter for the two-photon transition is $\eta=0.044$ at an axial trapping frequency of $\omega_{z}=2 \pi \times 872 \mathrm{kHz}$. Thus, after Doppler cooling the ion resides within the Lamb-Dicke regime and Doppler broadening can be neglected.

The Rydberg-excitation lasers are sent through hydrogen-loaded, single-mode photonic crystal fibers. Such fibers offer laser mode cleanup and stable beam pointing while resisting ultraviolet solarization [37]. The lasers are focused by two commercial achromat lenses that image the $10-\mu \mathrm{m}$-diameter fiber core with unity magnification onto the ion. The laser beams are sent through holes in the endcap electrodes and up to $120 \mathrm{nW}(16.8 \mathrm{~mW})$ of $243 \mathrm{~nm}$ 
(309 $\mathrm{nm})$ laser light is focused to $(5.1 \pm 0.8) \mu \mathrm{m}$ [(6.8 \pm 1.7$) \mu \mathrm{m}$ horizontally, $(4.9 \pm 1.0) \mu \mathrm{m}$ vertically] beam waist at the position of the ion.

\section{APPENDIX B: ION TRAP}

The ion is confined in a macroscopic linear Paul trap with titanium electrodes in a sapphire mount. The trap consists of four blade electrodes for radial confinement and two end-cap electrodes with optical access holes for axial confinement. Since Rydberg atoms may be extremely sensitive to electric fields (polarizability $\mathcal{P}_{n} \sim n^{7}$ ), with $n$ the principal quantum number of the Rydberg state, the ions are confined very close to the electric quadrupole null to minimize any detrimental effects of the fields to the stability of Rydberg ions. Using the "cross-correlation" and the "resolved sideband" techniques [38,39], micromotion is minimized and the residual electric field at the position of the ion is estimated to be $\left(3_{-3}^{+12}\right) \mathrm{Vm}^{-1}$. The trap electrodes are electroplated in gold (work function $\approx 5.3 \mathrm{eV}$ ) to avoid the emission of photoelectrons if ultraviolet laser light hits the electrode surfaces (243-nm photons carry $5.1 \mathrm{eV}$ energy). Photoelectron emission causes time-varying stray electric fields and thus worsens the residual electric field at the ion position. With the gold coating, the micromotion compensation parameters do not change over weeks of working with the ultraviolet Rydberg-excitation lasers.

\section{APPENDIX C: STATE PREPARATION AND DETECTION OF A SINGLE RYDBERG RESONANCE}

A single isolated Rydberg resonance is accessible in the Rydberg excitation from the initial state $4 D_{5 / 2}$ to the Rydberg state $42 S_{1 / 2}$. This excitation scheme is interesting from a quantum information perspective, since $4 D_{5 / 2}$ may be used together with the $5 S_{1 / 2}$ ground state to store an optical qubit. The Rydberg excitation could be used for entanglement operations between two such optical qubits.

For state preparation, a Doppler- (or sideband-)cooled ion is initialized in the $4 D_{5 / 2} m_{J}=-\frac{5}{2}$ Zeeman sublevel. First, any population in $4 D_{5 / 2}$ is returned to the ground state using the 1033-nm "repump" laser and the ion is Doppler cooled. The 674-nm "qubit" laser, which drives the $5 S_{1 / 2} \leftrightarrow 4 D_{5 / 2}$ transition, has a narrow linewidth $(<2 \pi \times 600 \mathrm{~Hz})$, which allows transitions between specific Zeeman sublevels to be individually addressed. By alternately driving the $5 S_{1 / 2} m_{J}=+\frac{1}{2} \leftrightarrow 4 D_{5 / 2} m_{J}=-\frac{3}{2}$ transition and removing population from $4 D_{5 / 2}$ using the 1033-nm repump laser, population may be optically pumped to the $5 S_{1 / 2} m_{J}=-\frac{1}{2}$ Zeeman sublevel. Next, population is transferred from the initial $5 S_{1 / 2}$ Zeeman sublevel to a specific $4 D_{5 / 2}$ Zeeman sublevel using the narrow 674-nm qubit laser. The fluorescence detection lasers are then turned on to check whether the population transfer was successful.

The detection of successful Rydberg excitation from initial state $4 D_{5 / 2}$ is simpler than for $4 D_{3 / 2}$, as the initial state $4 D_{5 / 2}$ can be directly distinguished from the final state $5 S_{1 / 2}$ by fluorescence detection without any need for additional shelving pulses. The Rydberg excitation and detection sequence is as follows. (i) Both Rydberg lasers are turned on, coupling $4 D_{5 / 2} m_{J}=-\frac{5}{2}$ via the intermediate state $6 P_{3 / 2} m_{J}=-\frac{3}{2}$ to $42 S_{1 / 2} m_{J}=-\frac{1}{2}$. According to quantum defect theory calculations, $95 \%$ of the population in $42 S_{1 / 2}$ quickly decays to the $5 S_{1 / 2}$ ground state. (ii) Finally, fluorescence detection is used to distinguish between successful Rydberg excitations (population in $5 S_{1 / 2} \rightarrow$ fluorescence) and cases with no Rydberg excitation (population in $4 D_{5 / 2} \rightarrow$ no fluorescence).

\section{APPENDIX D: LASER-ION INTERACTION HAMILTONIAN}

The Hamiltonian used to simulate the laser-induced twophoton transition to Rydberg $S$ and $D$ states including the magnetic-field-induced Zeeman effect is [19-21]

$$
\begin{aligned}
H & =H_{e}+H_{e Q}(\mathbf{r}, t)+H_{B}+H_{L}, \\
H_{e} & =\sum_{\mathbf{L}} \varepsilon_{\mathbf{L}}|\mathbf{L}\rangle\langle\mathbf{L}|, \\
H_{B} & =-\frac{e}{2 m_{e} c}\left|B_{z}\right|\left(L_{z}+2 S_{z}\right), \\
H_{L} & =\sum_{j}-e \mathbf{r} \cdot \hat{\epsilon}_{j} E_{j} \cos \left(\mathbf{k}_{j} \cdot \mathbf{R}-\omega_{j} t\right),
\end{aligned}
$$

where $H_{e}, H_{e Q}(\mathbf{r}, t)=-e \Phi(\mathbf{r}, t), H_{B}$, and $H_{L}$ stand for the Hamiltonian for the valence electron, Rydberg electronquadrupole field coupling, Zeeman effect, and laserelectron interaction. In the Hamiltonian, $\mathbf{r}$ and $m_{e}$ are the position and mass of the electron, $\mathbf{R}$ the center-of-mass position of the ion, and $\mathbf{L}$ the multi-index quantum number $\mathbf{L}=\left\{n, L, J, m_{J}\right\} . \varepsilon_{\mathbf{L}}$ is the energy in the electronic state $|\mathbf{L}\rangle . e$ and $c$ are the elementary charge and speed of light in vacuum. $B_{z}$ is a static magnetic field parallel to the $z$ axis. $S_{z}$ and $L_{z}$ are the $z$ components of the spin and angular momentum operators. $E_{j}$ and $\hat{\epsilon}_{j}$ are the electric field and polarization of the $j$ th laser, whose wave vector and frequency are $\mathbf{k}_{j}$ and $\omega_{j}$.

In the experiment, the first laser couples low-lying states $\left|4 D \frac{3}{2} m_{J}\right\rangle$ (denoted by $\left|g_{m_{J}}\right\rangle$ ) and $\left|6 P \frac{1}{2} m_{J}\right\rangle$ (denoted by $\left.\left|e_{m_{J}}\right\rangle\right)$ and the second laser couples the state $\left|6 P \frac{1}{2} m_{J}\right\rangle$ and Rydberg state $\left|R_{m_{J}}\right\rangle$. We neglect the sideband transitions, as the two lasers are counterpropagating and the effective Lamb-Dicke parameter $\eta<0.1$. Using the relevant 
electronic states as bases, the laser-ion interaction can be expressed as

$$
\begin{aligned}
H_{L}= & \sum_{\mathbf{M}}\left[\hbar \Omega_{1, \mathbf{M}} \cos \omega_{1} t\left|e_{m_{J}}\right\rangle\left\langle g_{m_{J}^{\prime}}\right|\right. \\
& \left.+\hbar \Omega_{2, \mathbf{M}} \cos \omega_{2} t\left|R_{m_{J}}\right\rangle\left\langle e_{m_{J}^{\prime}}\right|+\text { H.c. }\right]
\end{aligned}
$$

where $\mathbf{M}=\left\{m_{J}, m_{J}^{\prime}\right\}$ is a two-index number, and Rabi frequencies $\Omega_{1, \mathbf{M}}=-e E_{1}\left\langle e_{m_{J}}\left|\mathbf{r} \cdot \hat{\epsilon}_{1}\right| g_{m_{J}^{\prime}}\right\rangle / \hbar$ and $\Omega_{2, \mathbf{M}}=$ $-e E_{2}\left\langle R_{m_{J}}\left|\mathbf{r} \cdot \hat{\epsilon}_{2}\right| e_{m_{J}^{\prime}}\right\rangle / \hbar$ depend on respective electronic states and laser polarization.

We point out that both micromotion and the trap-fieldmediated Rydberg electron center-of-mass coupling are relatively weak $[19,20,28]$. Both effects are not experimentally resolved for low-lying Rydberg states $n<30$, as polarizability and temperature of the ion are small, and micromotion is carefully compensated. Therefore, we do not consider these effects in the numerical simulation. However, the Rydberg electron center-of-mass coupling is observed for the higher-lying Rydberg state $42 S_{1 / 2}$, as we discuss in Sec. V.

\section{APPENDIX E: SPECTRA OF THE RYDBERG $24 D_{3 / 2}$ STATE}

Here, we provide a simple theory to explain the spectra shown in Fig. 4(a). First, we note that electronic states are completely specified once laser polarizations are given. This allows us to omit the labeling of the quantum number $m_{J}$ in the electronic low-lying states. The two Rydberg states that are coupled by the quadrupole fields are labeled by $\left|R_{1}\right\rangle$ and $\left|R_{2}\right\rangle$ for convenience. Upon applying rotatingwave approximations to the laser-induced transitions, the Hamiltonian to describe the Rydberg excitation dynamics [see Eq. (D1)] becomes

$$
\begin{aligned}
H_{D}= & \hbar \Delta_{e}|e\rangle\left\langle e\left|+\sum_{j} \hbar \Delta_{j}\right| R_{j}\right\rangle\left\langle R_{j}\right|+H_{\mathrm{rf}} \\
& +\frac{\hbar}{2}\left[\Omega_{l}|e\rangle\left\langle g\left|+\Omega_{u}\right| R_{1}\right\rangle\langle e|+\text { H.c. }\right]
\end{aligned}
$$

where we assume that the polarization is chosen such that state $|e\rangle$ couples to Rydberg state $\left|R_{1}\right\rangle . \quad \Delta_{e}=$ $\left(E_{e}-E_{g}\right) / \hbar-\omega_{1}$ and $\Delta_{j}=\left(E_{j}-E_{g}\right) / \hbar-\omega_{1}-\omega_{2}$ $(j=1,2)$ give detuning of the electronic transition with respect to the laser frequencies, where the energy $E_{s}=$ $\varepsilon_{s}+E_{s}^{(B)}(s=g, e, 1,2)$ takes into account both the electronic energy $\varepsilon_{s}$ and Zeeman shift $E_{s}^{(B)}$.

As $\left|\Delta_{e}\right|$ is typically larger than other quantities in the Hamiltonian, we can adiabatically eliminate state $|e\rangle$, which yields

$$
\begin{aligned}
H_{D} \approx & -\frac{\hbar \Omega_{l}^{2}}{4 \Delta_{e}}|g\rangle\left\langle g\left|+\hbar\left(\Delta_{1}-\frac{\Omega_{u}^{2}}{4 \Delta_{e}}\right)\right| R_{1}\right\rangle\left\langle R_{1}\right| \\
& +\hbar \Delta_{2}\left|R_{2}\right\rangle\left\langle R_{2}\right|+\frac{\hbar}{2}\left[\Omega^{\prime}\left|R_{1}\right\rangle\langle g|+\text { H.c. }\right] \\
& +H_{\mathrm{rf}},
\end{aligned}
$$

where $\Omega^{\prime}=-\Omega_{l} \Omega_{u} / 2 \Delta_{e}$ is the two-photon Rabi frequency. Using the experimental parameters, we find that $\Omega_{u}^{2} / 4 \Delta_{e} \sim$ $2 \pi \times 10 \mathrm{MHz}$ and $\Omega^{\prime} \sim 2 \pi \times 10 \mathrm{kHz}$ while $\left|\Omega_{l}^{2} / 4 \Delta_{e}\right|$ is in the sub-kHz range, which can be neglected.

We proceed by expanding the Rydberg states in terms of Floquet states $\left|R_{j}, k\right\rangle(k=0, \pm 1, \ldots)$, where $k$ denotes quadrupolar excitations of the rf field. To explain the main peaks in Fig. 4, we need to take into account only transitions $\left|R_{1}, 0\right\rangle \rightarrow\left|R_{2}, \pm 1\right\rangle$, i.e., by absorbing or emitting one quadrupolar rf excitation. Equation (E2) becomes

$H_{D} \approx \hbar \Delta_{1} \sum_{j}\left|R_{j}\right\rangle\left\langle R_{j}\right|+\frac{\hbar}{2}\left[\Omega^{\prime}\left|R_{1}\right\rangle\langle g|+\right.$ H.c. $]+H_{F}$,

where

$$
\begin{aligned}
H_{F}= & -\frac{\hbar \Omega_{u}^{2}}{4 \Delta_{e}}\left|R_{1}\right\rangle\left\langle R_{1}\left|+\left(E_{2}^{(B)}-E_{1}^{(B)}\right)\right| R_{2}\right\rangle\left\langle R_{2}\right| \\
& +\sum_{k} k \hbar \Omega\left|R_{2}, k \Omega\right\rangle\left\langle R_{2}, k \Omega\right| \\
& +\frac{\hbar C}{2}\left[\left|R_{2}, \Omega\right\rangle\left\langle R_{1}|+| R_{2},-\Omega\right\rangle\left\langle R_{1}\right|+\text { H.c. }\right] .
\end{aligned}
$$

The Hamiltonian $H_{F}$ is the key result. The eigenenergy of $H_{F}$ determines the peaks shown in Fig. 4(a). One example for the $\sigma^{-} / \sigma^{+}$transition is given in Sec. IV.

[1] D. J. Wineland, C. Monroe, W. M. Itano, D. Leibfried, B. E. King, and D. M. Meekhof, Experimental Issues in Coherent Quantum-State Manipulation of Trapped Atomic Ions, J. Res. Natl. Inst. Stand. Technol. 103, 259 (1998).

[2] C. J. Ballance, T. P. Harty, N. M. Linke, M. A. Sepiol, and D. M. Lucas, High-Fidelity Quantum Logic Gates Using Trapped-Ion Hyperfine Qubits, Phys. Rev. Lett. 117, 060504 (2016).

[3] J. P. Gaebler, T. R. Tan, Y. Lin, Y. Wan, R. Bowler, A. C. Keith, S. Glancy, K. Coakley, E. Knill, D. Leibfried, and D. J. Wineland, High-Fidelity Universal Gate Set for ${ }^{9} \mathrm{Be}^{+}$ Ion Qubits, Phys. Rev. Lett. 117, 060505 (2016).

[4] T. Monz, P. Schindler, J. T. Barreiro, M. Chwalla, D. Nigg, W. A. Coish, M. Harlander, W. Hänsel, M. Hennrich, and R. Blatt, 14-Qubit Entanglement: Creation and Coherence, Phys. Rev. Lett. 106, 130506 (2011).

[5] J. Chiaverini, J. Britton, D. Leibfried, E. Krill, M. D. Barrett, R. B. Blakestad, W. P. Itano, J. D. Jost, C. Langer, R. Ozeri, T. Schaetz, and D. J. Wineland, Implementation of 
the Semiclassical Quantum Fourier Transform in a Scalable System, Science 308, 997 (2005).

[6] K. A. Brickman, P. C. Haljan, P. J. Lee, M. Acton, L. Deslauriers, and C. Monroe, Implementation of Grover's Quantum Search Algorithm in a Scalable System, Phys. Rev. A 72, 050306 (2005).

[7] S. Debnath, N. M. Linke, C. Figgatt, K. A. Landsman, K. Wright, and C. Monroe, Demonstration of a Small Programmable Quantum Computer with Atomic Qubits, Nature (London) 536, 63 (2016).

[8] T. Monz, D. Nigg, E. A. Martinez, M. F. Brandl, P. Schindler, R. Rines, S. X. Wang, I. L. Chuang, and R. Blatt, Realization of a Scalable Shor Algorithm, Science 351, 1068 (2016).

[9] J. Chiaverini, D. Leibfried, T. Schaetz, M. D. Barrett, R. B. Blakestad, J. Britton, W. M. Itano, J. D. Jost, E. Knill, C. Langer, R. Ozeri, and D. J. Wineland, Realization of Quantum Error Correction, Nature (London) 432, 602 (2004).

[10] P. Schindler, J. T. Barreiro, T. Monz, V. Nebendahl, D. Nigg, M. Chwalla, M. Hennrich, and R. Blatt, Experimental Repetitive Quantum Error Correction, Science 332, 1059 (2011).

[11] D. Nigg, M. Müller, E. A. Martinez, P. Schindler, M. Hennrich, T. Monz, M. A. Martin-Delgado, and R. Blatt, Quantum Computations on a Topologically Encoded Qubit, Science 345, 302 (2014).

[12] A. Friedenauer, H. Schmitz, J. T. Glueckert, D. Porras, and T. Schaetz, Simulating a Quantum Magnet with Trapped Ions, Nat. Phys. 4, 757 (2008).

[13] J. T. Barreiro, M. Müller, P. Schindler, D. Nigg, T. Monz, M. Chwalla, M. Hennrich, C. F. Roos, P. Zoller, and R. Blatt, An Open-System Quantum Simulator with Trapped Ions, Nature (London) 470, 486 (2011).

[14] B. P. Lanyon, C. Hempel, D. Nigg, M. Müller, R. Gerritsma, F. Zähringer, P. Schindler, J. T. Barreiro, M. Rambach, G. Kirchmair, M. Hennrich, P. Zoller, R. Blatt, and C. F. Roos, Universal Digital Quantum Simulation with Trapped Ions, Science 334, 57 (2011).

[15] R. Islam, C. Senko, W. C. Campbell, S. Korenblit, J. Smith, A. Lee, E. E. Edwards, C.-C. J. Wang, J. K. Freericks, and C. Monroe, Emergence and Frustration of Magnetism with Variable-Range Interactions in a Quantum Simulator, Science 340, 583 (2013).

[16] J. I. Cirac and P. Zoller, Quantum Computations with Cold Trapped Ions, Phys. Rev. Lett. 74, 4091 (1995).

[17] D. Kielpinski, C. Monroe, and D. J. Wineland, Architecture for a Large-Scale Ion-Trap Quantum Computer, Nature (London) 417, 709 (2002).

[18] C. Monroe, R. Raussendorf, A. Ruthven, K. R. Brown, P. Maunz, L.-M. Duan, and J. Kim, Large-Scale Modular Quantum-Computer Architecture with Atomic Memory and Photonic Interconnects, Phys. Rev. A 89, 022317 (2014).

[19] M. Müller, L. Liang, I. Lesanovsky, and P. Zoller, Trapped Rydberg Ions: From Spin Chains to Fast Quantum Gates, New J. Phys. 10, 093009 (2008).

[20] F. Schmidt-Kaler, T. Feldker, D. Kolbe, J. Walz, M. Müller, P. Zoller, W. Li, and I. Lesanovsky, Rydberg Excitation of
Trapped Cold Ions: A Detailed Case Study, New J. Phys. 13, 075014 (2011).

[21] W. Li and I. Lesanovsky, Entangling Quantum Gate in Trapped Ions via Rydberg Blockade, Appl. Phys. B 114, 37 (2014).

[22] D. Jaksch, J. I. Cirac, P. Zoller, S. L. Rolston, R. Côté, and M. D. Lukin, Fast Quantum Gates for Neutral Atoms, Phys. Rev. Lett. 85, 2208 (2000).

[23] T. Wilk, A. Gaëtan, C. Evellin, J. Wolters, Y. Miroshnychenko, P. Grangier, and A. Browaeys, Entanglement of Two Individual Neutral Atoms Using Rydberg Blockade, Phys. Rev. Lett. 104, 010502 (2010).

[24] L. Isenhower, E. Urban, X. L. Zhang, A. T. Gill, T. Henage, T. A. Johnson, T. G. Walker, and M. Saffman, Demonstration of a Neutral Atom Controlled-NOT Quantum Gate, Phys. Rev. Lett. 104, 010503 (2010).

[25] H. Labuhn, D. Barredo, S. Ravets, S. de Léséleuc, T. Macrì, T. Lahaye, and A. Browaeys, Tunable TwoDimensional Arrays of Single Rydberg Atoms for Realizing Quantum Ising Models, Nature (London) 534, 667 (2016).

[26] T. Feldker, P. Bachor, M. Stappel, D. Kolbe, R. Gerritsma, J. Walz, and F. Schmidt-Kaler, Rydberg Excitation of a Single Trapped Ion, Phys. Rev. Lett. 115, 173001 (2015).

[27] P. Bachor, T. Feldker, J. Walz, and F. Schmidt-Kaler, Addressing Single Trapped Ions for Rydberg Quantum Logic, J. Phys. B 49, 154004 (2016).

[28] W. Li and I. Lesanovsky, Electronically Excited cold Ion Crystals, Phys. Rev. Lett. 108, 023003 (2012).

[29] H. Friedrich, Theoretical Atomic Physics, 3rd ed. (Springer, Berlin, Heidelberg, New York, 2006).

[30] V. Lange, M. A. Khan, U. Eichmann, and W. Sandner, Rydberg States of the Strontium Ion, Z. Phys. D 18, 319 (1991).

[31] M. Aymar, C. H. Greene, and E. Luc-Koenig, Multichannel Rydberg Spectroscopy of Complex Atoms, Rev. Mod. Phys. 68, 1015 (1996).

[32] W. Li, A. W. Glaetzle, R. Nath, and I. Lesanovsky, Parallel Execution of Quantum Gates in a Long Linear Ion Chain via Rydberg Mode Shaping, Phys. Rev. A 87, 052304 (2013).

[33] R. Nath, M. Dalmonte, A. W. Glaetzle, P. Zoller, F. Schmidt-Kaler, and R. Gerritsma, Hexagonal Plaquette Spin-Spin Interactions and Quantum Magnetism in a Two-Dimensional Ion Crystal, New J. Phys. 17, 065018 (2015).

[34] N. Kolachevsky, J. Alnis, S. D. Bergeson, and T. W. Hänsch, Compact Solid-State Laser Source for $1 s-2 s$ Spectroscopy in Atomic Hydrogen, Phys. Rev. A 73, 021801 (2006).

[35] A. Wilson, C. Ospelkaus, A. VanDevender, J. Mlynek, K. Brown, D. Leibfried, and D. Wineland, A 750-mW, Continuous-Wave, Solid-State Laser Source at $313 \mathrm{~nm}$ for Cooling and Manipulating Trapped ${ }^{9} \mathrm{Be}^{+}$Ions, Appl. Phys. B 105, 741 (2011).

[36] H.-Y. Lo, J. Alonso, D. Kienzler, B. C. Keitch, L. E. de Clercq, V. Negnevitsky, and J. P. Home, All-Solid-State Continuous-Wave Laser Systems for Ionization, Cooling 
and Quantum State Manipulation of Beryllium Ions, Appl. Phys. B 114, 17 (2014).

[37] Y. Colombe, D. H. Slichter, A. C. Wilson, D. Leibfried, and D. J. Wineland, Single-Mode Optical Fiber for High-Power, Low-Loss UV Transmission, Opt. Express 22, 19783 (2014).
[38] D. J. Berkeland, J. D. Miller, J. C. Bergquist, W. M. Itano, and D. J. Wineland, Minimization of Ion Micromotion in a Paul Trap, J. Appl. Phys. 83, 5025 (1998).

[39] J. Keller, H. L. Partner, T. Burgermeister, and T. E. Mehlstäubler, Precise Determination of Micromotion for TrappedIon Optical Clocks, J. Appl. Phys. 118, 104501 (2015). 